\title{
Management of musculoskeletal dysfunction in infants (Review)
}

\author{
DAN YAO, XINGQIANG DENG and MINGGUANG WANG \\ Department of Neurology, Xuzhou Children's Hospital, Xuzhou, Jiangsu 221002, P.R. China
}

Received November 13, 2015; Accepted April 4, 2016

DOI: $10.3892 / \mathrm{etm} .2016 .3226$

\begin{abstract}
Excessive crying (or infant colic) is a common pain syndrome of infancy without any specific known aetiology or effective management. Many cases result in long-term poor sleep, behavioral problems and parental stress. The biomechanical aspects of this condition lack adequate investigation despite its strong link with assisted and/or difficult births. The present review focused on the current trends in the management of this mal-musculoskeletal health of infants associated with the condition of excessive crying. In addition, the risk factors associated with therapeutic procedures used to manage the above conditions were also discussed.
\end{abstract}

\section{Contents}

1. Introduction

2. Proposed aetiologies

3. Clinical management

4. Mechanism of manual treatment

5. Risk implications

\section{Introduction}

Musculoskeletal maladaptation in infants is defined as the failure of the motor system to respond, or to respond aberrantly to appropriate sensory stimuli due to biomechanical fault $(1,2)$. Normal response patterns are generated when the musculoskeletal system responds appropriately to the central nervous system, which mediates sensorimotor integration of the environment as well as physiological demands. When the musculoskeletal system cannot respond to normal signals due to biomechanical compromise, the infant's response to signals may be inappropriate, inefficient, ineffective or aberrant. These responses become characterised as functional problems of

Correspondence to: $\mathrm{Dr}$ Xingqiang Deng, Department of Neurology, Xuzhou Children's Hospital, 18 Sudibei Road, Xuzhou, Jiangsu 221002, P.R. China

E-mail: dengxingqiang361@163.com

Key words: musculoskeletal dysfunction, infants, crying syndrome, chiropractic manual therapy infancy and most commonly include excessive crying, inefficient sleep habits and sometimes ineffective feeding (3).

Musculoskeletal pain is the most common reason for the referral of children to rheumatologists (4). Investigators suggested that spinally mediated reflexes caused by tactile stimulation causing pain sensations are heightened in neonates due to lower thresholds (5). Behavioral reaction to pain is often used to evaluate the infant's discomfort, however it cannot be considered accurate primarily because facial reactions develop slowly, advancing over time and age. Therefore, neonate and young infants may demonstrate a reduced physical reaction to pain, despite having an exaggerated physiological response (5). Infant pain is a specialised topic because infants are pre-verbal and because of clinical difficulty in assessing and measuring pain and acknowledging that infants experience pain without apparent or obvious injury (6). Even physiological measurements such as heart, respiratory rates and blood pressure may not be useful in this age group, particularly in persistent pain or discomfort (7). Observation of infant behaviors of facial expression, crying, body movements, sleep patterns and inconsolability have been found useful but may also be indicative of a stress response and not of pain. Understanding of infant pain is limited in part due to ethical issues that limit invasive investigations in children (8), and prohibit study of the above concepts in human infants. The present review aimed to enlighten the management avenues available for the above chronic problem of young infants. The risk factors associated with these management avenues were also assessed.

\section{Proposed aetiologies}

If aetiology is established, then effective treatment may be identified and employed. It has been suggested that infant colic can be treated by changes in feeding and is influenced by cow milk protein intolerance (CMPI) (9). As such, there are cases of excessive crying that are indeed caused by an allergy or sensitivity to cow milk. However, if that is the case, the aetiology and treatment are simple and clear, i.e., remove the offending substance (from the mother's diet) or, if formula fed, switch the child to a hydrolysed formula or an amino acid formula in order that the offending milk protein cannot cause the irritation (10). However, if the cause and treatment are simple and clear, then the child's affliction can be easily diagnosed as CMPI and, therefore, is NOT colic or inconsolable crying of infancy (11). Further dietary changes, such as increased fibre or lactase, are not considered useful (12). Although $70 \%$ of the adult population has primary lactase deficiency, this generally 
first occurs at the age of 5-6 years and is extremely unlikely in infancy (13). Although dietary interventions such as the introduction of lactase are attempted, there is little evidence that any formulations have indicated any long-term positive effect on the condition (14). It is widely agreed that there is no association between method (breast or bottle) of feeding and infant colic (15).

Children with excessive crying should undergo complete assessment to be certain there is no disease process causing the problem. However, since evidence indicates that $<5 \%$ have a medical problem that precipitates the crying (16), this creates ambiguity, with an excessively crying infant, fearful parents, and an insufficient medical diagnosis. Although the clinical diagnosis is unclear, the presenting picture is clear. The excessively crying infant appears healthy, thriving and growing. The inconsolable crying starts at 8-14 days of age (17) and episodes of crying are most common at the end of the day (18). Excessive crying was originally defined as that which lasted $>3 \mathrm{~h} /$ day, $>3$ days/week for $>3$ weeks. Diagnoses based on time of crying have little utility and more modern definitions accept that parents can report accurately whether or not their child suffers from colic, and this is known as the 'mother's diagnosis' (19). However, whether these are actual features of the disorder or of other aetiologies (such as general pain) remains to be clarified.

\section{Clinical management}

Different types of therapy have been utilized to relieve this serious issue of musculoskeletal dysfunction including hospitalization, pharmacology, behavioral interventions, homeopathy, naturopathic interventions, herbal formulation, dietary interventions and manual medicine. However, those modalities resulted in expense but little relief for the families (20). Although 1 of 6 children present to the hospital with a severe crying episode, hospitalization is not considered appropriate and may even lead to harmful or unnecessary interventions (21). Similarly, pharmacological treatments, such as Dicyclomine (an anticholinergic drug) are considered harmful (22), with severe adverse effects including respiratory difficulties, seizure and death. Consequently this drug has been removed from the repertoire of treatments prior to 6 months of age. The most common allopathic medication administered, Simethicone, has been shown in three double-blind studies to have no effect (22). Other medications, including Gaviscon, proton-pump inhibitors and acid suppressants, routinely prescribed for gastroesophageal reflux disease are commonly given to this age group for excessive crying despite lack of evidence to support their use (23).

Emerging evidence suggests that probiotics used to modulate microbiotic intestinal flora in the child may be useful. However, additional investigations are necessary to determine its actual efficacy in the pain syndrome of excessive crying (24) and for general health. Other ingested therapeutics such as herbal medicine, homeopathy, botanical supplements and teas have been subjected to few trials and have produced mixed results, raising concerns with regard to adverse effects (25). There also concerns regarding their potential to interfere with infant feeding (26). A wide variety of behavioral interventions including car ride simulation, enhanced carrying, uniform daily care and swaddling (27) have been investigated with insufficient evidence to recommend. On the other hand, chiropractic manual therapy in the treatment of infant colic has shown some promise (28). Additionally, there may be some, albeit limited, evidence for the use of massage in the first 6 months (29). The first time that manual medicine was reported as a treatment for infant colic was by Still in 1910 (30). Since then, parents have preferred chiropractic care in increasing numbers (31). Although positive results from the manual treatment of infant colic have been reported, the exact mechanism responsible for such results remains to be elucidated (32).

\section{Mechanism of manual treatment}

There is a potentially plausible biological mechanism by which chiropractic (manual) therapy may be successful. It is known that pain sensations occur in infants and that the sensory system is intact well before birth and increases in ability to process sensations shortly after birth (33). Pain sensations are most likely amplified in the extremely young ages and this affects short-term (8) and long-term functional movement patterns (34). If the infant is subject to tense muscles and imbalances in the spine and ligaments, it is reasonable to postulate that chiropractic manual therapy may alleviate the imbalance, restore normal musculoskeletal function and relieve tension and pain. This is achieved through mild finger pressure to a restrictive barrier. The rationale for this type of care is the hypothesis that well-being is associated with connective tissues, including muscles and ligaments and fascia, working smoothly together with the skeletal structure along with the nervous system to integrate and relieve misalignments and decrease soft-tissue tensions in the body. This process may enhance blood and nerve supply to any areas of dysfunction, thus decreasing pain sensations. In adults chiropractic care has been shown to reduce pain (35).

Chiropractic is manual medicine and is one of the most common types of manual therapies that include osteopathy and physiotherapy (36). Chiropractors are musculoskeletalists who differ from the other types of manual therapists in that chiropractors are trained as primary contact clinicians and are responsible for diagnosis along with specific type of manual therapies (37). As such, it is a profession dedicated to the prevention and treatment of musculoskeletal problems of humans of all ages. Chiropractors focus on the mechanical stress of the human body and this begins from the time of intra-uterine life and includes passage through the birth canal (38) as well as everyday life stresses that can manifest as pain, loss of function and even disability.

Chiropractic therapy is demarcated by the use of manual treatment of joints and soft tissues intended to promote the health and well-being of the patient. It may encompass a wide variety of techniques or modalities including chiropractic manual therapy, light touch (hold and release), moderate touch or deeper pressure involving treatment of trigger points, myofascial release, neuromuscular techniques or massage (39). Touch is the first sense to develop in humans and therapeutic touch therapy has been known and used in health care since the time of Hippocrates or before ( 460 to $370 \mathrm{BC})$ and continues to be in use for pain control in modern medicine for even premature neonates (40). Significant benefits of 
chiropractic manual therapy for pain control have been documented in at least one meta-analysis (41). In Europe, 52\% of parents seek complementary and alternative medicine for their children (42) and many more seek conventional medical assistance worldwide.

\section{Risk implications}

Although there are a limited number of studies focused on whether chiropractic therapy for infants is safe, current evidence has shown few adverse events from care of the paediatric patient, only transient irritability from infant care and one (uncertain) adverse event from colic treatment (43). When placed into the context of $\geq 30$ million chiropractic treatments of children annually in the USA alone, adverse events may be deemed extremely rare. Chiropractors in the United Kingdom and most of the rest of Europe adhere to the Chiropractic Reporting and Learning System of adverse events or near miss reporting and no serious adverse events from infant or child treatment have occurred since its inception in 2006 (44). Although it has been claimed that adverse effects resulting from the chiropractic care of paediatric patients may be under-reported (45), it is unlikely that parents are to stand by and watch a child being injured or in pain without complaint. Any magnitude of the problem would certainly be associated with malpractice claims, which are virtually non-existent (46). There are no guidelines that systematise either the investigation or treatment of pain syndromes of infancy at this time. A medical guideline is a protocol constructed to guide decision making around evidence-based criteria regarding diagnosis, management and treatment in specific areas of health care (47). These have been used throughout the history of health care, although in early times were based on tradition or authority opinion. Pain syndromes of infancy including the most common problem, excessive crying of infancy provide a window of opportunity into investigation of the musculoskeletal health of infants.

In conclusion, based on the above literature, it can be concluded that manual chiropractic therapy is the most successful option for the management of paediatric musculoskeletal health of infants. In addition, few adverse effects have been reported, and these are negligible in comparison to the beneficial accounts of manual therapy. However, additional investigations need to be performed to elucidate the core mechanisms responsible for existing results of manual therapy.

\section{References}

1. Reher C, Kuny KD, Pantalitschka T, Urschitz MS and Poets CF: Randomised crossover trial of different postural interventions on bradycardia and intermittent hypoxia in preterm infants. Arch Dis Child Fetal Neonatal Ed 93: F289-F291, 2008.

2. Section J, Gibbons SD, Barton T, Greenberg DE, Jo CH and Copley LA: Microbiological culture methods for pediatric musculoskeletal infection: A guideline for optimal use. J Bone Joint Surg Am 97: 441-449, 2015.

3. St James-Roberts I: Infant crying and sleeping: Helping parents to prevent and manage problems. Prim Care 35: 547-567, viii, 2008.

4. McGhee JL, Burks FN, Sheckels JL and Jarvis JN: Identifying children with chronic arthritis based on chief complaints: Absence of predictive value for musculoskeletal pain as an indicator of rheumatic disease in children. Pediatrics 110: 354-359, 2002.
5. Fitzgerald $M$ and Beggs S: The neurobiology of pain: Developmental aspects. Neuroscientist 7: 246-257, 2001.

6. Finley AG, Franck LS, Grunau R and von Baeyer CL: Why Children's Pain Matters. Int Assoc Study Pain Task Force Acute Pain XIII: 1-2, 2005.

7. de Jesus JA, Tristao RM, Storm H, Da RA and Campos DJ: Heart rate, oxygen saturation, and skin conductance: A comparison study of acute pain in Brazilian newborns. Conf Proc IEEE Eng Med Biol Soc 2011: 1875-1879, 2011.

8. Fitzgerald M and Walker SM: Infant pain management: A developmental neurobiological approach. Nat Clin Pract Neurol 5: 35-50, 2009.

9. Lothe L and Lindberg T: Cow's milk whey protein elicits symptoms of infantile colic in colicky formula-fed infants: A double-blind crossover study. Pediatrics 83: 262-266, 1989.

10. Plaza-Martin AM: [Food allergies in paediatrics: Current concepts]. An Pediatr (Barc) Mar 82016 (In Spanish). (Epub ahead of print)

11. Miller $\mathbf{J}$ and Weber-Hellstenius. Is infant colic an allergic response to cow's milk? What is the evidence? J Clin Chiropr Pediatr 14: 1097-1102, 2013.

12. Hall B, Chesters J and Robinson A: Infantile colic: A systematic review of medical and conventional therapies. J Paediatr Child Health 48: 128-137, 2012.

13. Heyman MB; Committee on Nutrition: Lactose intolerance in infants, children, and adolescents. Pediatrics 118: 1279-1286, 2006.

14. Dupont C, Rivero M, Grillon C, Belaroussi N, Kalindjian A and Marin V: Alpha-lactalbumin-enriched and probioticsupplemented infant formula in infants with colic: Growth and gastrointestinal tolerance. Eur J Clin Nutr 64: 765-767, 2010.

15. Lucas A and St James-Roberts I: Crying, fussing and colic behaviour in breast-and bottle-fed infants. Early Hum Dev 53: 9-18, 1998.

16. Freedman SB, Al-Harthy $\mathrm{N}$ and Thull-Freedman J: The crying infant: Diagnostic testing and frequency of serious underlying disease. Pediatrics 123: 841-848, 2009.

17. Paradise JL: Maternal and other factors in the etiology of infantile colic. Report of a prospective study of 146 infants. JAMA 197: 191-199, 1966.

18. Soltis J: The signal functions of early infant crying. Behav Brain Sci 27: 443-490, 2004.

19. Miller J, Newell D and Bolton J: Efficacy of manual therapy in infant colic: A pragmatic single-blind, randomised controlled trial. Arch Dis Child 97 (Suppl 1): A114, 2012.

20. Crouch JL, Skowronski JJ, Milner JS and Harris B: Parental responses to infant crying: The influence of child physical abuse risk and hostile priming. Child Abuse Negl 32: 702-710, 2008.

21. Savino F, Pelle E, Castagno E, Palumeri E and Oggero R: Must infants with colic really be hospitalized? Acta Paediatr 96: 1109, author reply 1110, 2007.

22. Garrison MM and Christakis DA: A systematic review of treatments for infant colic. Pediatrics 106: 184-190, 2000.

23. Savino F and Castagno E: Overprescription of antireflux medications for infants with regurgitation. Pediatrics 121: 1070, author reply $1070-1071,2008$.

24. Savino F, Cordisco L, Tarasco V, Palumeri E, Calabrese R, Oggero R, Roos S and Matteuzzi D: Lactobacillus reuteri DSM 17938 in infantile colic: A randomized, double-blind, placebocontrolled trial. Pediatrics 126: e526-e533, 2010.

25. Thompson EA, Bishop JL and Northstone K: The use of homeopathic products in childhood: Data generated over 8.5 years from the Avon Longitudinal Study of Parents and Children (ALSPAC). J Altern Complement Med 16: 69-79, 2010.

26. Zhang Y, Fein EB and Fein SB: Feeding of dietary botanical supplements and teas to infants in the United States. Pediatrics 127: 1060-1066, 2011.

27. McRury JM and Zolotor AJ: A randomized, controlled trial of a behavioral intervention to reduce crying among infants. J Am Board Fam Med 23: 315-322, 2010.

28. Dobson D: The effectiveness of chiropractic in the treatment of infantile colic - a multicentre randomised controlled trial: a study protocol. Southampton University, 2013.

29. Underdown A, Barlow J, Chung V and Stewart-Brown S: Massage intervention for promoting mental and physical health in infants aged under six months. Cochrane Database Syst Rev 18: CD005038, 2006.

30. Still AT: Osteopathy Research and Practice. Kirksville, MO: Self-published; 1910. 
31. Barnes PM, Bloom B and Nahin RL: Complementary and alternative medicine use among adults and children: United States, 2007. Natl Health Stat Report 10: 1-23, 2008.

32. Hayden C and Mullinger B: A preliminary assessment of the impact of cranial osteopathy for the relief of infantile colic Complement Ther Clin Pract 12: 83-90, 2006.

33. Buonocore $G$ and Bellieni $C$ : Neonatal pain and oxidative stress. Minerva Pediatr 62 (Suppl 1): 59-60, 2010.

34. Hermann C, Hohmeister J, Demirakça S, Zohsel K and Flor H: Long-term alteration of pain sensitivity in school-aged children with early pain experiences. Pain 125: 278-285, 2006.

35. Shi Q, Langer G, Cohen J, Cleeland CS and Charles S: People in pain: How do they seek relief? J Pain 8: 624-636, 2007.

36. Owen G: Investigation into Research Methods used for Manual Therapies in Paediatrics. Anglo European College of Chiropractic/Bournemouth University, Bournemouth, 2013.

37. World Health Organization: Guidelines on Chiropractic. WHO Press, Geneva, Switzerland, 2005.

38. Stellwagen L, Hubbard E, Chambers C and Jones KL: Torticollis, facial asymmetry and plagiocephaly in normal newborns. Arch Dis Child 93: 827-831, 2008.

39. Goertz CM, Long CR, Hondras MA, Petri R, Delgado R, Lawrence DJ, Owens EF and Meeker WC: Adding chiropractic manipulative therapy to standard medical care for patients with acute low back pain: Results of a pragmatic randomized comparative effectiveness study. Spine (Phila Pa 1976) 38: 627-634, 2013.

40. Honda N, Ohgi S, Wada N, Loo KK, Hagashimoto Y and Fukuda K: Effect of therapeutic touch on brain activation of preterm infants in response to sensory punctate stimulus: A nearinfrarered spectropscopy-based study. Arch Dis Child Fetal Neonatal Ed 98: F244-F248, 2013.
41. Moyer CA, Rounds J and Hannum JW: A meta-analysis of massage therapy research. Psychol Bull 130: 3-18, 2004.

42. Zuzak TJ, Boňkováb J, Caredduc D, Garamid M, Hadjipanayise A, Jazbec J, Merrick J and Miller J: Published data and expert perspectives on the use of complementary and alternative medicine by children in Europe. J Altern Complement Med 1: S34-S47, 2013.

43. Wilson PM, Greiner MV and Duma EM: Posterior rib fractures in a young infant who received chiropractic care. Pediatrics 130: e1359-e1362, 2012.

44. Wangler M, Zaugg B, Peterson C, Thiel H and Finch RP: Reflecting on safety in the management of chiropractic patients. In: ECU Convention Proceedings May 16-18; Amsterdam, pp170-172, 2012

45. Vohra S, Johnston BC, Cramer K and Humphreys K: Adverse events associated with pediatric spinal manipulation: A systematic review. Pediatrics 119: e275-e283, 2007.

46. Shaw L, Descarreaux M, Bryans R, Duranleau M, Marcoux H, Potter B, Ruegg R, Watkin R and White E: A systematic review of chiropractic management of adults with Whiplash-Associated Disorders: Recommendations for advancing evidence-based practice and research. Work 35: 369-394, 2010.

47. Sutton D, Gross DP, Cote P, Randhawa K, Yu H, Wong JJ, Stern P, Varatharajan S, Southerst D, Shearer HM, et al: Multimodal care for the management of musculoskeletal disorders of the elbow, forearm, wrist and hand: A systematic review by the Ontario Protocol for Traffic Injury Management (OPTIMa) Collaboration. Chiropr Man Therap 24: 8, 2016. 